\title{
Dress-up: Deep Neural Framework for Image-based Human Appearance Transfer
}

This paper was downloaded from TechRxiv (https://www.techrxiv.org).

\section{LICENSE}

CC BY 4.0

SUBMISSION DATE / POSTED DATE

$12-01-2022$ / 14-01-2022

\section{CITATION}

Ghodhbani, Hajer; NEJI, MOHAMED; Qahtani, Abdulrahman M.; Almutiry, Omar; dhahri, habib; Alimi, Adel (2022): Dress-up: Deep Neural Framework for Image-based Human Appearance Transfer. TechRxiv. Preprint. https://doi.org/10.36227/techrxiv.18211424.v1

$\mathrm{DOI}$ 


\title{
Dress-up: Deep Neural Framework for Image-based Human Appearance Transfer
}

\author{
Hajer Ghodhbani ${ }^{\mathrm{a},}$, , Mohamed Neji ${ }^{\mathrm{a}, \mathrm{b}}$, Abdulrahman M. Qahtani ${ }^{\mathrm{c}}$, Omar Almutiry $^{\mathrm{d}}$, Habib Dhahri ${ }^{\mathrm{d}}$, Adel M. Alimi ${ }^{\mathrm{a}, \mathrm{e}}$ \\ ${ }^{a}$ REsearch Groups in Intelligent Machines (REGIM Lab), University of Sfax, National Engineering School of Sfax (ENIS), BP 1173, Sfax, 3038, Tunisia \\ ${ }^{b}$ National School of Electronics and Telecommunications of Sfax Technopark, BP 1163, CP 3018 Sfax, Tunisia \\ ${ }^{c}$ Department of Computer Science, College of Computers and Information Technology, Taif University, P.O.Box. 11099, Taif 21944, Saudi Arabia \\ ${ }^{d}$ College of Applied Computer Science, King Saud University, Riyadh, Saudi Arabia \\ ${ }^{e}$ Department of Electrical and Electronic Engineering Science, Faculty of Engineering and the Built Environment, University of Johannesburg, South Africa
}

\section{ABSTRACT}

\section{Keywords:}

Artificial Intelligence

Fashion industry

Huma appearance transfer

Virtual try-on

Segmentation
The fashion industry is at the brink of radical transformation. The emergence of Artificial Intelligence (AI) in fashion applications creates many opportunities for this industry. Interesting to this matter, we proposed a flexible person generation system for virtual try-on, presented in this paper, and aiming to treat the task of human appearance transfer across images while preserving texture details and structural coherence of the generated outfit. This challenging task has drawn increasing attention and made huge development of intelligent fashion applications. However, this task requires different challenges, especially, in the case of a wide divergences between the source and target images. To solve this problem, we proposed a flexible person generation framework called Dress-up to treat the 2D virtual try-on task. Dress-up is an end-to-end generation pipeline with three modules based on the task of imageto-image translation aiming to sequentially interchange garments between images, and produce dressing effects not achievable by existing works. The core idea of our solution is to explicitly encode the body pose and the target clothes by a pre-processing module based on the segmentation process. Then, a conditional adversarial network is implemented to generate target segmentation feeding to the alignment and translation networks to generate the final output. Our experimental results on the widely used DeepFashion dataset demonstrate that Dress-up provides significant results over the state-of-the-art methods on the subject of output quality despite different interactions of garments, and handles the virtual try-on task with a weakly supervised manner.

\section{Introduction}

Since 2020, everything was changed around the world, when the coronavirus pandemic sent its shockwaves in each country. These two years will go down in history as one of the most challenging and worst period on record for various sectors such as the fashion industry that finding itself in mid of unprecedented adversity which marked a drop in sales and a change in customer behavior. Last year and according to McKinsey Global Fashion Index analysis, fashion companies post approximately a $90 \%$ decline of their economic profit, after an increase of $4 \%$ in 2019 [1]. In the coming years and due to the doubt of the epidemiological situation around the world, the predictions for fashion apparel performance are related to different scenarios.

Despite the expectations that the coming period will be critical for fashion apparel, it will also be a period of investment to make changes in this area. The future opportunities will be created for companies that are able to adopt new solutions for digital shopping which is the main driver of fashion industry development in next years. Thus, yet the several impacts of this pandemic, fashion companies must innovate new ways and strategies to compete.
The digital opportunity in e-commerce is the most cited one by far, even in case of eradicating this pandemic. Last year, the online fashion industry growth marked higher anticipations compared to 2019 [1]. Thus, the current year suggest the beginning of novel chapter for the global fashion apparel based on lessons learned from last period which have been the key of huge change. The most important idea taken from all these events is that, similar to various industries, the fashion apparel, will leave this crisis in a distinct form than that in which it entered. Brands are driven to do all they could to recover their lost revenue. So, they need to find new strategies to motivate customers to purchase online. They should adapt to the faster change by adopting new working practices that have emerged from the crisis and trying to elevate the online customer experience with advanced methods. In coming years and according to fashion leaders, it will be a greatest growth in e-commerce due to the improvement of digital customer experience through the use of artificial intelligence.

One of these improvements is to allow consumers purchasing fashion items after fitting them online like in real stores because the existing systems are unable to give the possibility for consumers to fit different garment items according to their desires. Therefore, fashion companies have to satisfy the preferences of

\footnotetext{
* Corresponding author.

E-mail address: hajer.ghodhbani@regim.usf.tn (H. Ghodhbani).
} 
their clients and engage them with customized purchasing experience to build confident purchase decisions. Additionally, allowing users to try-on fashion items online will not just boosting the shopping experience, but also increase fashion industries sales because these solution can play an important role to reduce return rates and improve customer satisfaction. Following this objective, our work focuses on using AI technology to develop an imagebased virtual fitting system.

A virtual fitting would be a way to see the virtual effects, but it is still far from solved due to the challenge to virtually change the texture and pattern of clothes deformation, especially, when we use an image-based approach to transfer clothes. To overcome this problem, we have proposed a flexible $2 \mathrm{D}$ person generation pipeline for virtual try-on based on Image-to-Image translation task (I2I). Recently, research in this task has been growing faster than ever before due to the appearance of deep generative models and their capacity to generate photorealistic images which is the case of our solution that will be detailed in this paper.

After an overview of related work in second section, we present, in the third section, the task of image based transfer and expose our problematic and the related challenges. Then, in section IV, we will describe our proposed system composed of three stage which will be edited separately. Firstly, we'll describe the pretreatment stage implemented to obtain the body segmentation and clothes segmentation used as an input to the warping stage based on generative network. Then, the texture stage will exposed to demonstrate how we are using I2I networks to deal with the task of texture generation. Section $\mathrm{V}$ will show the experiments process with illustration of the implemented scenario. The obtained results will be presented, in this same section, with different comparisons to show the functionalities allowed by our framework. Finally, the paper will conclude with a brief summary in the last section.

\section{Related work}

\subsection{Virtual try-on}

Image based virtual try-on task focuses on interchanging desired garment to a given human body, it is considered as a challenging task since it requires to preserve details and identity while transferring clothes from the image of such person to another in different poses. The simplest way to deal with try-on task is to replace a specific item of clothes with a target one [2-8].

With other works, the challenge become more complicated, the proposed virtual fitting system should be more closely by generating images with all the target garments [9-13]. Men et al. [9] implemented generative model called Attribute-decomposed GAN (ADGAN) to control image synthesis. The idea is to encode garment attribute of the input person image into a series of decomposed component codes and embed them into the latent space to build the full style code by feeding a concatenation of these codes into StyleGAN generator [14]. Another framework called Outfit-VITON [10] is proposed as an image-based virtual try-on approach dependent on splitting segmentation mask of garment image into various regions to be associated with encoded garments. This work is not able to change the pose of the target person which is ensured by our framework.

In last year, Sarkar et al. [11, 12] proposed an efficient photorealistic re-rendering system with high-quality results by adopting an explicit control over both style and pose for image synthesis. Before that, SwapNet [13] was proposed as one of the important virtual try-on framework based on conditioning network such as U-net [15] used to generate a mutually exclusive segmentation mask of the desired garment.
Our work falls within this objective by proposing a system able to realize the garment transfer between different images of persons. The most advantages of our work compared with the above methods are the possibility to allow transfer of all the garment item and not only such one garment in each class because our system is based on segmentation masks and not on garment classes; and also the high quality of generated results by preserving details and identity of target images. We are adopted the same process shown on SwapNet architecture [13], an end-toend pipeline with specific sub-networks for each level combined to ensure the garment transfer from one person's image onto different target pose.

\subsection{Image-to-Image Translation}

Image-to-image translation (I2I) task focus on transferring appearances or other specific information from source image to target image while maintaining the content representations. Recently, I2I has drawn great interest and made huge development due to the appearance of its wide range of applications in many computer vision and image processing problems. Generative adversarial networks (GAN) [16] is the most used method for I2I next to variational auto-encoders [17].

GANs have achieved a high-grade visual quality in different image processing problems such as conditional image synthesis [18-22] and style transfer [23-25]. In our work, we have interested to the conditional image synthesis to generate images conditioning on specific constraint task. The warp module of our architecture is inspired from the U-Net network [15] which is based on two conditioning images where the first provides the desired garment and the second present the target body.

Additionally, we have focused on another form of conditional image synthesis by using conditional semantic labels to synthesize images following the given category labels [26-29]. This method has a great advantage to add more diversity and control ability at the semantic segmentation to generate photorealistic images. In our texture module, we have used a combination between two conditioned network $[28,29]$ to achieve realistic results.

\section{Image-Based Transfer}

\subsection{Problem Statement}

Garment transfer task presents a crucial challenge because successful transfer requires significant structural changes to source images, and as introduced previously, directly interchange texture details of desired clothes to target body presents overburden results due to the poor quality of transfer process. Given the Fig.1 where $\mathrm{I}_{C}$ presented image of person with desired garment, and $\mathrm{I}_{\mathrm{B}}$, corresponding to person on target body, the large differences in body structure and clothes outlines between the input images makes the task of directly transferring clothes items from $\mathrm{I}_{\mathrm{C}}$ to $\mathrm{I}_{\mathrm{B}}$ very difficult. Thus and instead of this process, we suggest to transfer, firstly, the clothing segmentation $\mathrm{C}_{\text {seg }}$ of $\mathrm{I}_{\mathrm{C}}$, based on $\mathrm{B}_{\text {seg }}$ and the body segmentation of $\mathrm{I}_{\mathrm{B}}$, to generate the desired clothes segmentation $\mathrm{C}_{\text {seg. }}^{\prime}$. When $\mathrm{C}_{\text {seg }}^{\prime}$ is generated, we pass to the step of transferring the texture details from $\mathrm{I}_{\mathrm{C}}$ to $\mathrm{I}_{\mathrm{B}}$ conditioned on $\mathrm{C}^{\prime}{ }_{\text {seg }}$ to obtain final result $\mathrm{I}_{\mathrm{G}}$.

The proposed solution must treat the image translation task to generate photo-realistic images conditioning on specific inputs such as semantic segmentation. However, reaching high fidelity image translation with maintaining of high quality of generated images remains a big challenge due to the difficulty to control image styles and shapes. Thus, a typical system to ensure the image-based transfer task will be needed. 


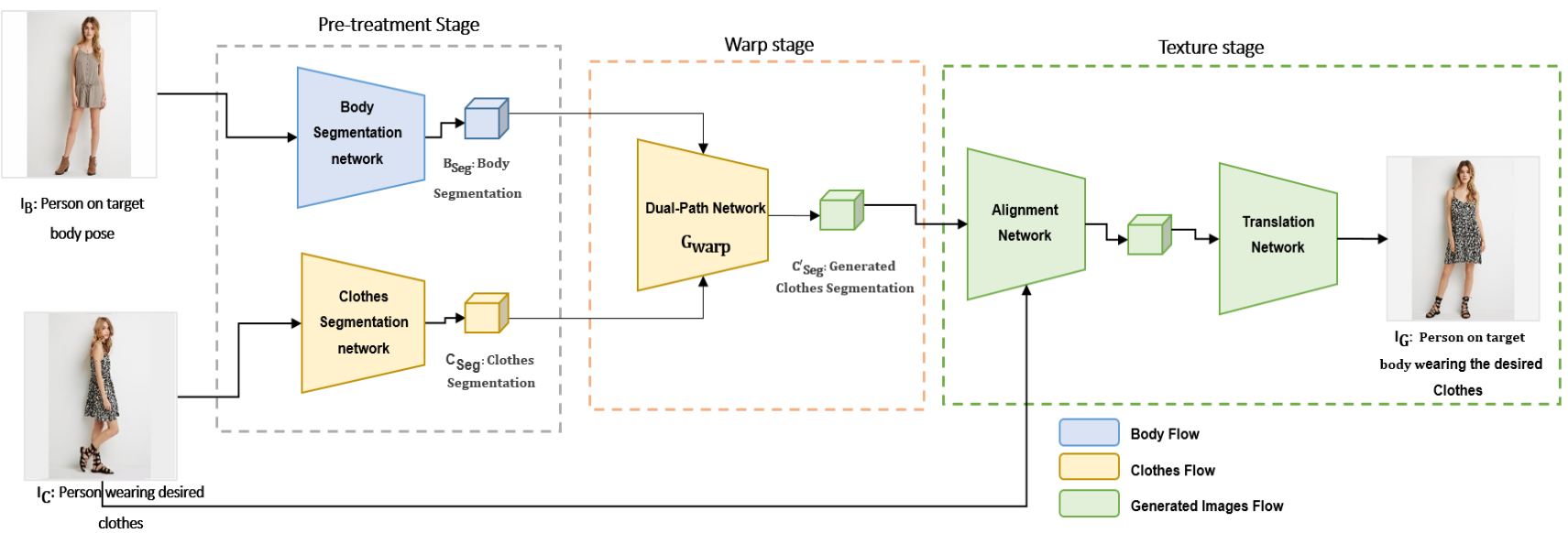

Fig.1. The architecture of our framework consists of three stages. (1) The pre-treatment stage implemented to pre-process the input data. (2) The warp stage aims to generate intermediary clothes representation consistent with the target body. (3) The texture stage synthesizes detailed according to the output of warp stage.

\subsection{Challenge}

Our work requires treating the challenge of jointly inferring the body pose and garment of person by solving three sub-problems. In our case, the adopted approach is to deal with the image content by transferring clothes information between input images. Firstly, the garment pieces must be identified from target clothes images. Then, the shape will be transferred across different poses. Finally, the clothes textures need to be synthesized in the target bodies with realistic details. A demonstration of clothing transfer results obtained with our work is presented in the following Fig.2.

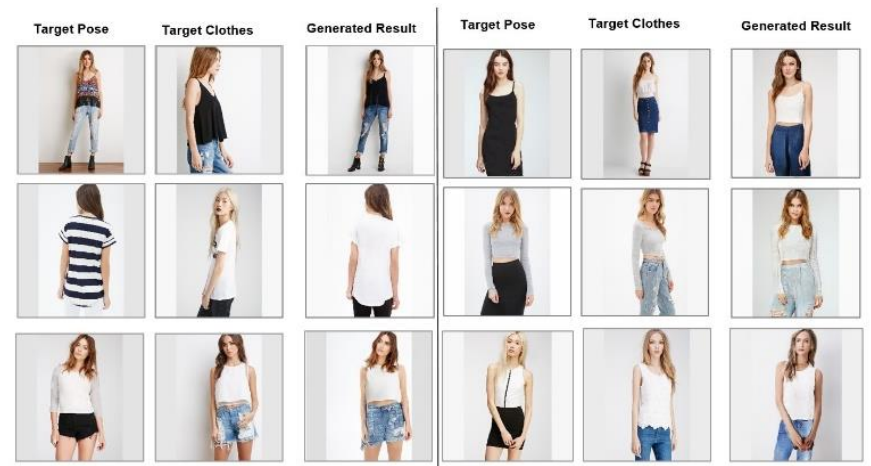

Fig.2. Demonstration of clothing transfer task: Dress-up allowing the transfer of garment appearance between two different images.

Therefore, our work focuses on garment interchange between pictures of different persons with no constraints on identity in the input and the output images. The clothing transfer is a challenging task requiring to disentangle the desired clothes from the corresponding person and retargeting it to a different body.

\section{Dress-up}

This section describes our garment transfer system called Dress-up illustrated in Fig.2, it is developed to interchange clothes between different views of images while maintaining details of target garment and identity of the target person. This challenging task is achieved by using three stages, to change either the person or the clothes and recombine them as we desire. The scenario is as follow, taking an image representing the person with target clothes and another image concerned the person in target body, we generate a final output showing a person wearing the desired clothes from the first input image but on the pose of the second one. Therefore, the functionalities of our system are as follow:
- Developing an image-based virtual fitting system to interchange garment appearances between pictures of persons with arbitrary pose.

- Transferring garment across images and maintain the picture's identity while transferring clothes details to target view.

- Synthesizing an image of a person wearing desired clothes from two different view images, more precisely.

- Encountering problems like degradation of details, occlusions, and physical dislocation and missing.

The proposed pipeline aim to treat the pose and clothes synthesis separately by providing garment and body segmentations obtained from based representation of desired clothes and target body. Therefore, we use, firstly, these segmentations to perform the target pose of $\mathrm{I}_{\mathrm{B}}$ but with the clothes in $\mathrm{I}_{C}$. The clothes segmentation of $\mathrm{I}_{C}$ and the body segmentation of $\mathrm{I}_{\mathrm{B}}$ are obtained in the pre-treatment stage by using two different methods [30, 31]. In the third module, we presented a texturing network that takes the synthesized clothing segmentation and the desired clothing images as inputs to generate the final result. The architecture of the proposed framework is illustrated in Fig. 2, and the description of each stage are detailed on the following sections.

\subsection{Pre-treatment Stage}

This stage preprocess data to obtain the two inputs required for training the model during the next stage (warp stage). These inputs $\left(\mathrm{C}_{\text {seg }}, \mathrm{B}_{\text {seg }}\right)$ are considered respectively, as a concise representation of desired clothes and target body used to ensure the desired shape change by generating the synthesized segmentation $\mathrm{C}_{\text {seg }}^{\prime}$, in the target body pose conditioning on the target clothes considered as the input of the texture stage and used to obtain final output $\mathrm{I}_{\mathrm{G}}$.

For the pre-processing phase, we have trained existing networks [30, 31] on DeepFashion dataset [32] which contains $800 \mathrm{~K}$ images of persons wearing different garment items, and extracting body and clothing representations from each input. In this step, we have followed the SwapNet work [13] which used the DeepFashion dataset to train the preprocessing networks LIP_SSL [33] and Unite-the People [34] for clothes and body segmentation respectively. For our architecture, we replaced these networks with other more suitable models used for the same purposes. These choice are demonstrated in the following sections. 


\subsubsection{Clothes Segmentation}

For clothes segmentation, we employed LIP-JppNet network [30] which is an updated version of LIP_SSL [33] with more accuracy on segmentation task. This network aims to associate into a single network different contexts such as the image-level context, the body joint and the part context and refined context (Fig.3). It treats different various challenging problems especially, shared feature extraction using ResNet-101 [35], then, pixel-wise label prediction and keypoint heatmap prediction with a part module and a joint module to capture the part context and keypoint context while generating parsing score maps and pose heatmaps. Finally, an iterative refinement is used to predict maps and generate context to obtain better results.

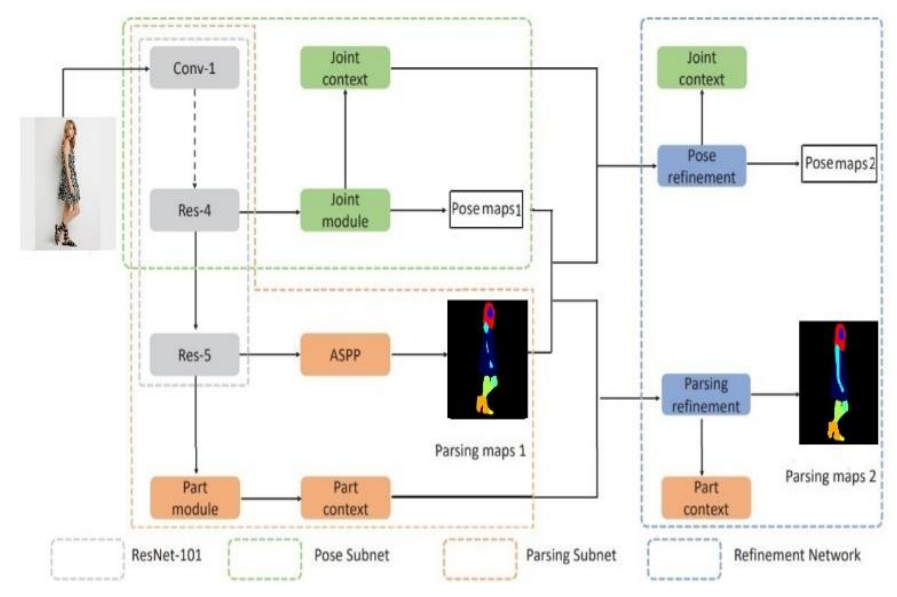

Fig.3. Architecture of LIP-JppNet network [30].

The SwapNet work [13] represents the clothing segmentation labels as 18-channel maps which means that this representation is consisting on eighteen different clothes labels (e.g. dress, pants, and denim). This representation allowing the model to warp each individual segment separately therefore we need high capacity to work with this network. In the other hand, by using LIP-Jpp-Net network, we have represented the desired clothing segmentation simply with 3-channel color-coded segmentation which can give sufficient information to generate the desired representation.

\subsubsection{Body Segmentation}

The second network is Neural Body Fitting (NBF) [31] with a standard semantic segmentation $\mathrm{CNN}$ into 12 semantic parts (Fig.3). An encoding CNN processes the semantic part probability maps to predict a Skinned Multi-person Linear body model parameters (SMPL) [36]. Then an SMPL implementation is used to obtain a projection of the pose-defining points to $2 \mathrm{D}$. With these points, a loss on $2 \mathrm{D}$ vertex positions can be back propagated through the entire model.

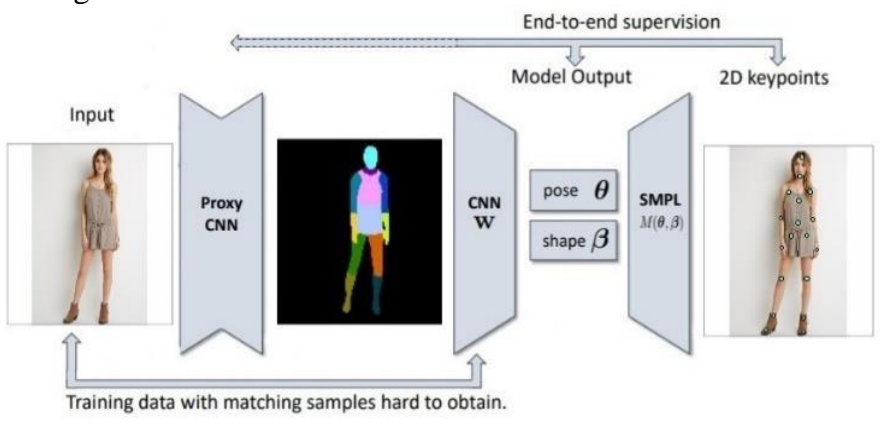

Fig.4. Architecture of NBF network [31]
In addition, $\mathrm{NBF}$ can treat $3 \mathrm{~d}$ pose by regressing the parameters of SMPL from a single image consisting of an intermediate $2 \mathrm{D}$ body part segmentation but in our case, we simply ignore this part and interesting only on obtaining the 2D representation. Therefore, it is easier to deal with NBF network and run it with a simple implementation which is not the case with Unite-the People used by SwapNet that working in real-time. Also, the inaccuracy of NBF on such results cannot cause much concern on generated results because it can be obtained with high quality even with noisy representation from the preprocessing models.

\subsection{Warp Stage}

The warp stage is the second part of our pipeline, which take as inputs the two outputs of the first stage $\left(\mathrm{C}_{\mathrm{seg}}, \mathrm{B}_{\mathrm{seg}}\right)$, respectively, the clothes segmentation and the body segmentation, to generate as output a target garment segmentation $\mathrm{C}_{\text {seg }}^{\prime}$ consistent with $\mathrm{C}_{\text {seg }}$, and strictly following the $\mathrm{B}_{\mathrm{seg}}$. This process is illustrated on Fig.5 which shows that this operation is based on a conditioned generative method, and precisely, on a dual path Unet network [15] to deal with the dual conditioning process consistent with the clothes and body segmentation. This network is composed of two encoders, one for the clothes and the other for the body, and a decoder to generate the final output by merging the two encoded representations. The generated segmentation $\mathrm{C}_{\text {seg }}^{\prime}$ is robustly conditioned on the body representation and weakly conditioned on the clothes representation.

In this stage, we are followed the SwapNet work [13] which aims to represent the generated clothes with segmentation mask. The process is as follow, the clothes encoder produces a feature map of size $512 \times 16 \times 16$ for each clothes representation $\mathrm{C}_{\text {seg }}$. In the other side, the body encoder produces also a feature map with the same size as $\mathrm{C}_{\text {seg }}(16 \times 16$ features of size 512$)$ to represent the desired body segmentation $B_{\text {seg }}$. The generated image is strongly conditioned on $\mathrm{B}_{\text {seg }}$ and weakly conditioned on $\mathrm{C}_{\text {seg }}$ by encoding it into a narrow representation of $2 \times 2 \times 1024$, and then up-sampling it to a feature map of demanded size. After obtaining these encoded feature maps, the step of concatenation is needed and ensured by using 4 residual blocks to get a target feature map that had to be up-sampled to generate the desired clothing segmentation $\mathrm{C}_{\text {seg }}^{\prime}$. This representation makes the model more flexible to warp each individual segment separately.

As mentioned above, we implemented the warp module as a dual-path U-net [15] following SwapNet framework. The warping generator $G_{\text {warp }}$ has an encoder-decoder architecture that synthesizes a new shape map $\mathrm{C}_{\text {seg }}^{\prime}$ conditioned on body representation $B_{\text {seg }}$ and clothes representation $C_{\text {seg }}$.

$$
\mathrm{C}_{\text {seg }}^{\prime}=\mathrm{G}_{\mathrm{warp}}\left(\mathrm{B}_{\mathrm{seg}}, \mathrm{C}_{\mathrm{seg}}\right)
$$

The warping module is trained with the combination of cross entropy loss and GAN loss. Specifically, the warping module has the following learning objectives:

$$
\begin{gathered}
\mathrm{L}_{\mathrm{CE}}=-\sum_{c=1}^{3} 1\left(C_{\text {seg }}(\mathrm{i}, \mathrm{j})=\mathrm{c}\right)\left(\log \left(G_{\text {warp }}(\mathrm{i}, \mathrm{j})\right)\right) \\
\mathrm{L}_{\mathrm{adv}}=E_{x \sim p\left(C_{\text {seg }}\right)}[\mathrm{D}(\mathrm{x})]+E_{z \sim p\left(f 1_{\text {enc }}\left(C_{\text {seg }}, B_{\text {seg }}\right)\right)}[1- \\
D\left(f 1_{\text {dec }}(z)\right)(3) \\
\mathrm{L}_{\text {warp }}=\mathrm{L}_{\mathrm{CE}}+\lambda_{\mathrm{adv}} \mathrm{L}_{\mathrm{adv}}
\end{gathered}
$$

where $\lambda_{\text {adv }} L_{\text {adv }}$ refers to the adversarial component of the loss and $f 1_{\text {enc }}$ and $f 1_{\text {dec }}$ are the encoder and decoder components of the warp module. 


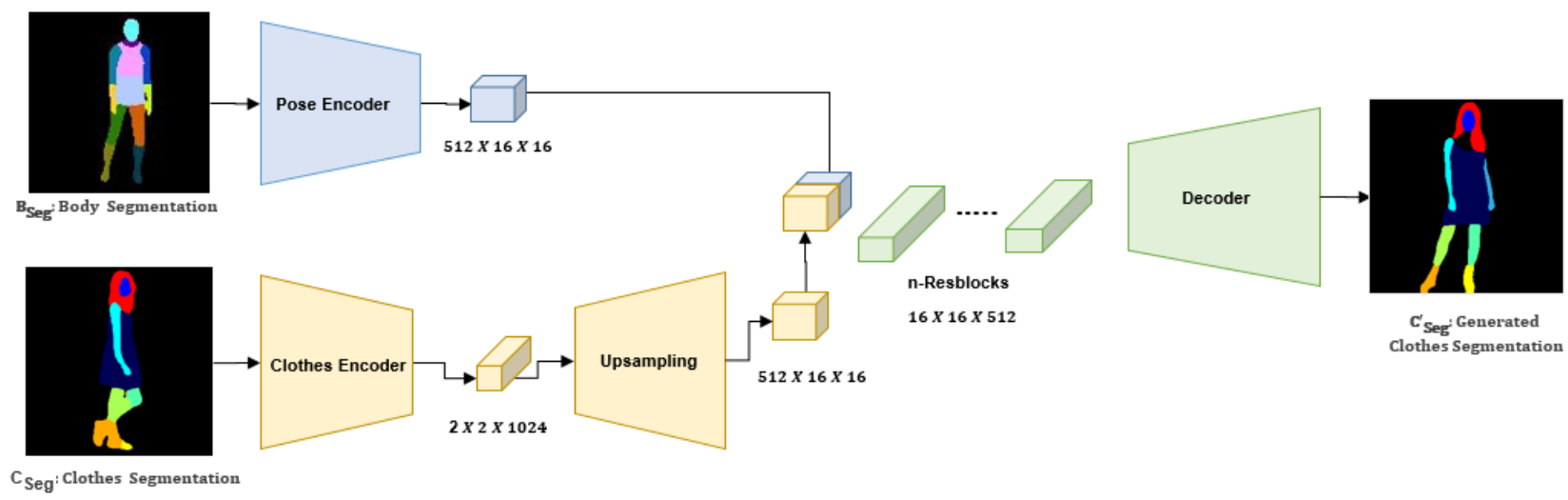

Fig.5. Architecture of warp stage based on the dual-path U-net network with two conditioning representations, one on the body segmentation and the other on clothes segmentation and inspired from SwapNet work [13].

\subsection{Texture Stage}

The third stage in our pipeline is, the texture module, aiming to generate texture details. The followed process consisting of using the target clothes segmentation $\boldsymbol{C}_{\text {seg }}^{\prime}$ to control the whole structure, and the image of person on target clothes to control texture generation. This module takes the generated clothes segmentation $\mathrm{C}_{\text {seg }}^{\prime}$ conditioned on body information $\mathrm{I}_{\mathrm{B}}$ and the reference clothes $\mathrm{I}_{\mathrm{C}}$, as inputs and synthesize an output $\boldsymbol{I}_{\boldsymbol{G}}$ of person on target body $\boldsymbol{I}_{\boldsymbol{B}}$ wearing the desired clothing on $\mathrm{I}_{\mathrm{C}}$. In other words, we aim to convert a segmentation mask to photorealistic image by extracting the high-level information of clothes representation and target style.

Similar to prior work [37], our texture stage consists of two essential parts working with an end-to-end manner: an alignment network and a translation network which are interconnected to synthesize realistic image from the semantic segmentation mask, and a reference style image (Fig. 6). The alignment network aligns the features of conditional inputs and reference style image and the translation network produces the final synthesis. The generated image contains the style in correspondence with the semantically objects in the reference image. Thus, the use of both the segmentation and image reference facilitate the task of translation with a weak supervised manner. The images are aligned to an intermediate representation to create the correspondence between the inputs and synthesize images according to the style image.

\subsubsection{Alignment Network}

The feature transport network aims to transport the feature of exemplars to be aligned with that of conditional inputs, thus providing accurate style guidance for the image translation. This network is inspired from the work of Zhang et al. [29] that used semantic correspondence to map the input images and create consistency representation of target style able to represent the semantics for both input images. As shown in Fig.6, we first adapt the target segmentation body $\mathrm{C}_{\text {seg }}$ and the image of target clothes $\mathrm{I}_{\mathrm{C}}$ to a shared style image. In other words, $\mathrm{C}_{\text {seg }}^{\prime}$ and $\mathrm{I}_{\mathrm{C}}$ are entered to the feature pyramid network that extracts feature maps which will be converted to the following adapted representations: $\mathrm{T}_{\mathrm{B}} \in$ $\boldsymbol{R}^{H W * C}$ and $\mathrm{T}_{\mathrm{C}} \in \boldsymbol{R}^{\boldsymbol{H} \boldsymbol{W * C}}$ (HW are feature spatial size; C is the channel-wise dimension).

These representations constitute the discriminative features that characterize the semantics of inputs. Thus, the alignment task presents an important step to realize the correspondence between different images. The features of $\mathrm{T}_{\mathrm{B}}$ and $\mathrm{T}_{\mathrm{C}}$ are matched with the correspondence layer proposed by the based work [29] in this task. Then, a correlation matrix $M \in \boldsymbol{R}^{\boldsymbol{H} \boldsymbol{W} * \boldsymbol{H} \boldsymbol{W}}$ is computed:

$$
M(u, v)=\frac{\widehat{\mathrm{T}_{\mathrm{B}}}(u)^{T} \widehat{\mathrm{T}}_{\mathrm{C}}(v)}{\left\|\widehat{T}_{\mathrm{B}}(\mathrm{u})\right\|\left\|\widehat{T}_{\mathrm{C}}(\mathrm{v})\right\|}
$$

Where $\widehat{\mathrm{T}_{\mathrm{B}}}(\mathrm{u})$ and $\widehat{\mathrm{T}_{\mathrm{C}}}(\mathrm{v}) \in R^{C}$ represent the channel-wise centralized feature of $\mathrm{T}_{\mathrm{B}}$ and $\mathrm{T}_{\mathrm{C}}$ in position $\mathrm{u}$ and $\mathrm{v} . \mathrm{M}(\mathrm{u}, \mathrm{v})$ indicates a higher semantic similarity between $\mathrm{T}_{B}(\mathrm{u})$ and $\mathrm{T}_{\mathrm{C}}(\mathrm{v})$.

The importance of this step resides on providing the easier way to the translation network to generate realistic results by referring to the correct regions in consistence with the reference clothes image $\mathrm{I}_{\mathrm{C}}$, which implicitly drives the model to learn the accurate correspondence. The correspondence style $\mathrm{I}_{\mathrm{S}}$ is generated according to $\mathrm{M}$ by selecting the most correlated pixels in $\mathrm{T}_{\mathrm{C}}$ and calculating their weighted average,

$$
\mathrm{I}_{\mathrm{S}}(\mathrm{u})=\sum_{v} \operatorname{softmax}_{v}(\alpha \mathrm{M}(\mathrm{u}, \mathrm{v})) \cdot \mathrm{T}_{\mathrm{C}}(\mathrm{v})
$$

Where, $\alpha$ is the coefficient that controls the sharpness of Softmax.

\subsubsection{Translation Network}

According to correspondent style image $\mathrm{I}_{\mathrm{S}}$ generated from the Alignment network, the translation network converts the constant code $\mathrm{z}$ to the final output $\mathrm{I}_{\mathrm{G}}$. With the aim to maintain the structural information of $\mathrm{I}_{\mathrm{S}}$, we used SPADE network [28] which has the ability to project the spatially adaptive style to different activation locations (Fig.6), and adopts a specific strategy of details generation by avoiding the normalization and feeding the input image through spatially adaptive modulation.

This network consisting of an adversarial trained encoderdecoder generator inspired by the idea of VAE [17] aiming to learn the mapping from a semantic representation to a reference image, and encoding its style into a latent style vector, from which the network generates output with desired style correspondent to reference image. The specificity of the spatially-adaptive normalization is its simple architecture with a simple layer used to synthesize photorealistic images by using reference representation as input. SPADE overcomes the lack of poor quality by taking the input layout through this layer and learned transformation to modulate the activations in normalization layers. The use of this network in texture stage demonstrate its advantage by allowing the control over style details.

Despite the great advantage of SPADE network to generate directly the desired appearance, there is an important point to mention about the style code which can only characterize the 


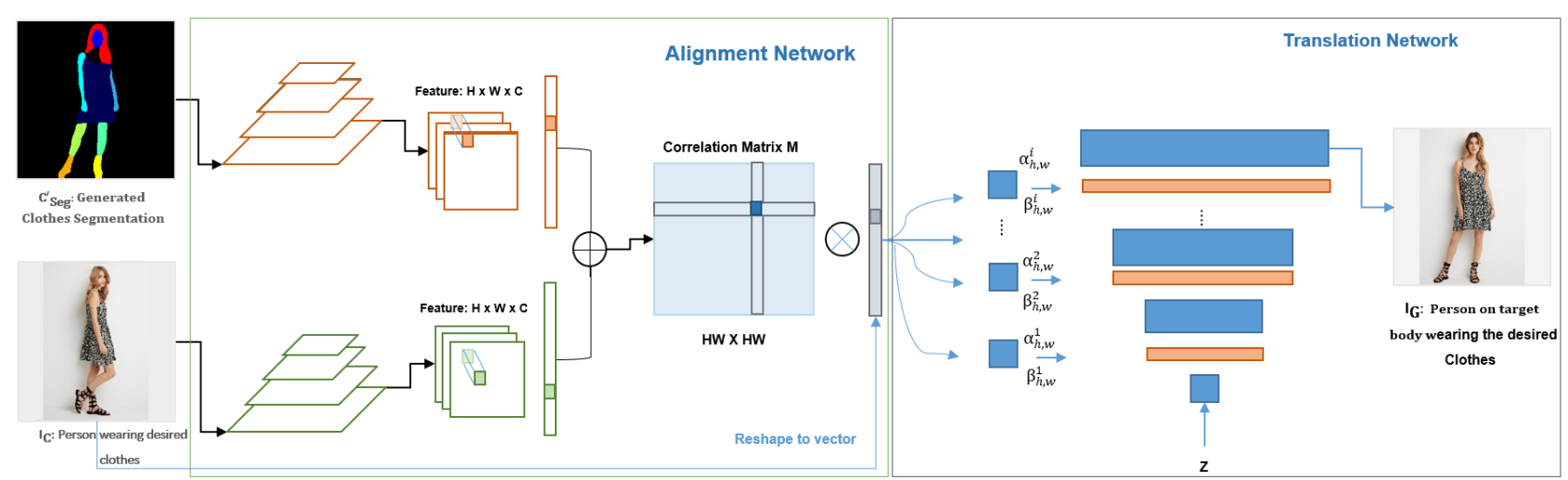

Fig.6. Texture stage architecture: The Alignment network takes the generated segmentation maps and target clothes as inputs, and generates a correspondence style that will be entered to the Translation network to obtain the image of person on target clothes.

global style of the target style image, whatever the relevant spatial information, and produces some local style "wash away" in generated image. For this reason, the built of alignment network as an intermediate step is required before using this network. Consequently, this step consisting in creating a correspondent style to the target image to guide the image translation by proposing an alignment network that transforms the inputs images to an intermediate representation to create the correspondence between them.

During training, the correspondent style image $\mathrm{I}_{\mathrm{S}}$ feeds into the SPADE layers, projected onto an embedding space, convolved to produce the modulation parameters $\alpha$ and $\beta$ which characterize the style of the clothes image, and mapped from $\mathrm{I}_{\mathrm{C}}$. Given the activation $F^{i} \in R^{\mathrm{C}_{\mathrm{i}} X \mathrm{H}_{\mathrm{i}} X \mathrm{~W}_{\mathrm{i}}}$ before the $i^{\text {th }}$ normalization layer, the reference style is injected through:

$$
\alpha_{h, w}^{i}\left(\mathrm{I}_{\mathrm{S}}\right) X \frac{\mathrm{F}_{c, h, w}^{i}-\mu_{h, w}^{i}}{\sigma_{h, w}^{i}}+\beta_{h, w}^{i}\left(\mathrm{I}_{\mathrm{S}}\right)
$$

where $\mu_{h, w}^{i}$ and $\sigma_{h, w}^{i}$ are the mean and standard deviation of activations and the symbols $\alpha_{h, w}^{i}, \beta_{h, w}^{i}$ to denote the functions that convert $\mathrm{m}$ to the scaling and bias values at the site $(\mathrm{h}, \mathrm{w})$ in the $i^{\text {th }}$ activation map.

At this stage, we used the adversarial loss by training a discriminator that discriminates the generated outputs and the real samples of target clothes. The translation network $G$ and the discriminator $\mathrm{D}$ are trained until the generation of images looking very similar to the real ones. Therefore, the adversarial objectives of $\mathrm{D}$ and $\mathrm{G}$ are respectively defined as:

$$
\begin{gathered}
L_{a d v}^{D}=-E\left[h\left(D\left(\mathrm{~T}_{\mathrm{C}}\right)\right)\right]-E\left[h\left(-D\left(G\left(\mathrm{~T}_{\mathrm{B}}, \mathrm{T}_{\mathrm{C}}\right)\right)\right]\right. \\
L_{a d v}^{G}=-E\left[D\left(G\left(\mathrm{~T}_{\mathrm{B}}, \mathrm{T}_{\mathrm{C}}\right)\right)\right]
\end{gathered}
$$

As a summary, the texture stage consisting of two related networks: i) Alignment Network that aim to convert the inputs from two different images to an intermediate feature images useful for obtaining correspondent style. ii) The translation network is based on the SPADE network [28] to generate the final result, employing the details of desired style from a generated style aligned semantically to the mask consisting on the estimated correspondence. These two networks work in a complementary way to ensure the objective of the texture stage that aim to generate photo-realistic images.

\section{Experiments}

In the experiments section, we present the scenario done by our framework, then, we expose the results of different modules of our architecture before presenting quantitative and qualitative comparisons with baselines and discussing our findings.

\subsection{Execution Scenario}

The scenario of our work is presented as an end-to-end pipeline to achieve three main functionalities. Firstly and as mentioned above, we train the Deep-Fashion dataset [32] to generate clothes segmentations to obtain, respectively, the body segmentation by using LIP-JppNet network [30] and NBF [31] (Fig.7.) Then, the segmentation representations obtained from the pretreatment stage will be an inputs to the warp stage of our framework, one image, showing the desired clothes $\mathrm{I}_{\mathrm{C}}$, and other $\mathrm{I}_{\mathrm{B}}$, presenting the target body. Firstly, we proceed to transfer the clothes segmentation of $\mathrm{I}_{\mathrm{C}}$, based on the body segmentation of $\mathrm{I}_{\mathrm{B}}$ to generate the target clothes segmentation $\mathrm{C}_{\text {seg }}^{\prime}$.

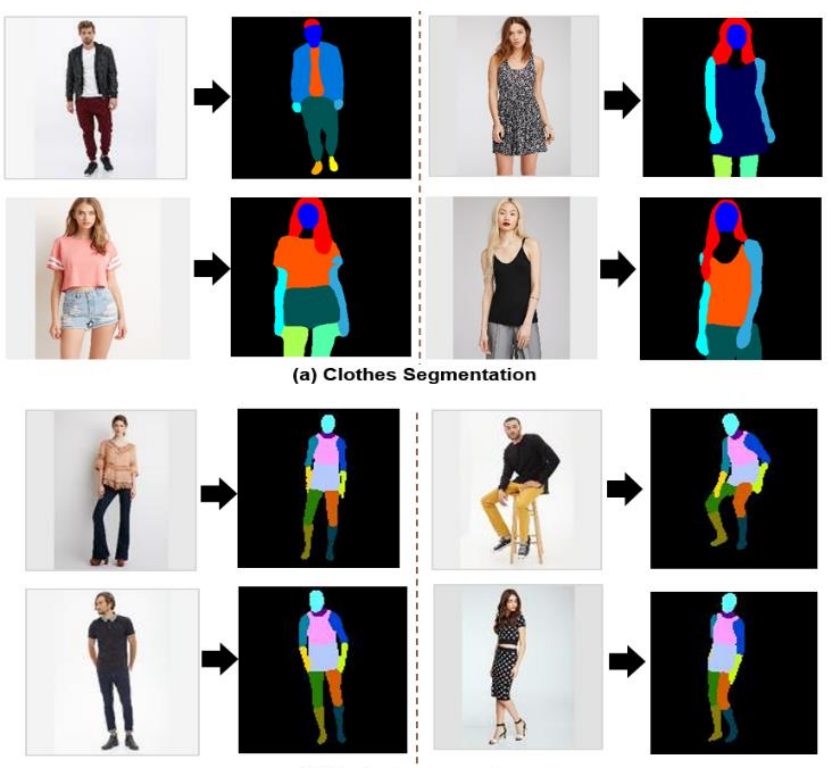

(b) Body Segmentation

Fig.7. Results of Pre-treatment stage that consists of two target segmentations: (a) body segmentation results, which are obtained by using NBF network [31] and (b) the clothes segmentation results generated by LIPJppNet network [30]. 
Also, to maintain the identity of target person before feeding it into texture stage, the face and hair segments must be replaced with appropriate segments in the clothes representation. Without this step, the target clothes will transferred in the same person instead of different persons. Finally and when the target clothes segmentation $\mathrm{C}_{\text {seg }}^{\prime}$ is obtained, we pass to transfer the garment details from $\mathrm{I}_{\mathrm{C}}$ to $\mathrm{I}_{\mathrm{B}}$ conditioned on $\mathrm{C}^{\prime}$ seg for final output. Fig.8 shows different obtained results.
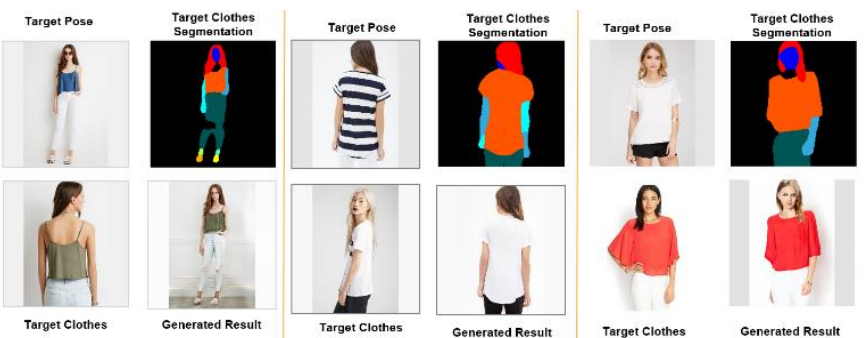

Fig.8. Example of generated results according to the based segmentation.

\subsection{Dataset}

We conduct experiments on the DeepFashion dataset [32] and precisely, the In-shop Clothes Retrieval Benchmark which containing 52,712 training images captured from fashion images of men and women models. We choose this dataset because it is the largest existing fashion dataset and it shows lots of diversities of various fashion images. Due to the difficulty to show different views of input images with similar clothes on distinct bodies, we have turned to the data augmentation technique.

\subsection{Baselines}

We compare our method with two leading image based transfer systems: the SwapNet [13] system, and the re-rendering system [11] to evaluate the performance of our proposed Dress-up framework on the garment transfer task. SwapNet is a GAN-based conditional image synthesis framework. The re-rendering uses a neural image-translation network. For comparison, we used the results provided by the authors on the DeepFashion dataset.

\subsection{Qualitative Evaluation}

We present qualitative comparisons of the competing methods (Fig.9 and Fig.10). We find that our method produces results with much better visual quality and fewer visible artifacts, especially for diverse scenes in the DeepFashion dataset. Our model is very close for generation details even in case of large pose deviation (e.g. interchange garment from a person in full body to a person in half body)

\subsubsection{Comparison with SwapNet}

In this section, we will make comparison of Dress-up with the work of Amit Raj et al. [13] called SwapNet, on the DeepFashion dataset (Fig.10), this work proposed a pipeline that interchanges clothes between two images of persons by resolving two sub-tasks: segmentation of input images to generated target map, then synthesis and transfer of clothes characteristics onto this generated representation. SwapNet has difficulty handling various pose changes and cannot hallucinate details for occluded regions. According to the comparison presented in Fig.9, our method overcomes these issues.

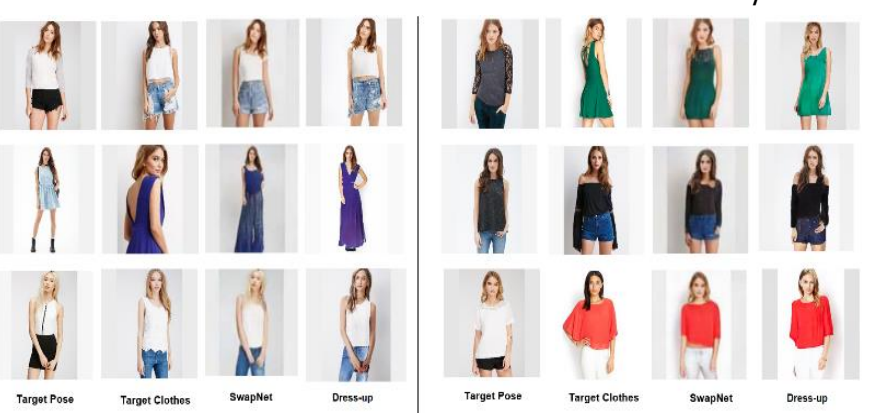

Fig.9. Comparison between Dress-up and SwapNet [13]

\subsubsection{Comparison with Re-Rendering}

We present additional comparison with Deep Re-rendering [11]. This work ensure the human image synthesis task by allowing the change of camera view, pose and clothes of the person in the source image. The authors suggest a highdimensional feature map to encode appearance as an intermediate representation translated within a generator network to realize rendering of the input person in a new pose. Despite the interesting results obtained with this work, our model shows more high details quality in the appearance of the clothes item (e.g. texture, color, shoes) which have been properly transferred onto the target body.

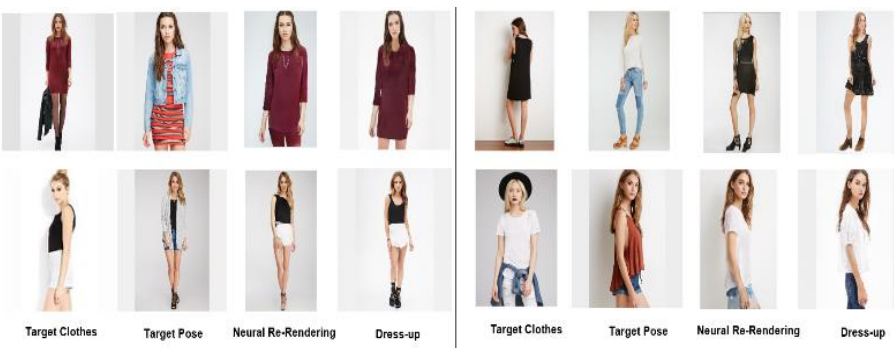

Fig.10. Comparison between Dress-up and Deep-Re-rendering [11] on the DeepFashion dataset.

\subsection{Quantitative Evaluation}

Quantitative results are presented in this section on different models using two popular measures, (i) Inception Score (IS) [38] and (ii) structural similarity (SSIM) [39], to evaluate the performance of our work and compare the quality of obtained results with baselines methods. Inception Score is a measure of how realistic images from a set look and how diverse they are. Higher IS indicates that produced images are diverse, and each one has a more meaningful object in it. On the other hand, SSIM computes the similarity between input and output images ranging from zero (dissimilarity) to one (similarity). We present the SSIM on a subset of data for which there are paired information.

Table 1. Quantitative results for different methods.

\begin{tabular}{lll} 
Model & SSIM & IS \\
\hline CGAN & 0.22 & 2.11 \\
\hline SwapNet & 0.83 & $3.04 \pm 0.052$ \\
\hline Ours (Dress-up) & $\mathbf{0 . 9 5}$ & $\mathbf{3 . 0 4} \pm \mathbf{0 . 1 5}$
\end{tabular}

According to table1, we observe that IS and SSIM metrics provide a clear proxy to measure performance and we can say that Dress-up performs the best in comparison with baselines models. 


\subsection{Effectiveness of Dress-up}

Dress-up has successfully solving wide pose changes between source and target images (Fig.9 and Fig.10). For example, when an input images contain a truncated body and other contains a full body, our model is able to generate high quality details. Furthermore, our framework is capable to manage various kinds of occlusions and generate images with high quality details. The second row in Fig.10 shows that the framework is, in some cases, unable to hold self-occlusion like fats. The representation of pose, such as body segmentation, provides better guidance to the reference body. Additionally, we demonstrate the convenience of adopting clothes segmentation as an intermediate representation (Fig.8). In cases where the generated clothes is inappropriate, we can edit the intermediate representation to better fit the clothes.

\section{Conclusion}

Artificial intelligence (AI) played a great role in boosting conversion on fashion industry, giving consumers the possibility to experiment the virtual fitting solutions without the integration of human interaction. In this context, we present Dress-up, a framework for single view garment transfer to meet the need for a novel approach to improve the traditional "end-to-end" training pipeline by highlighting the use of intermediary representations for the body and the texture style to realize garment transfer with photorealistic outputs.

\section{Acknowledgments}

We deeply acknowledge Taif University for Supporting this study through Taif University Researchers Supporting Project number (TURSP-2020/327), Taif University, Taif, Saudi Arabia. The research leading to these results has received funding from the Ministry of Higher Education and Scientific Research of Tunisia under the grant agreement number LR11ES48.

\section{References}

[1] C. d. C. de Bogota, et al., The state of fashion 2021 ' (2021).

[2] H. Dong, X. Liang, X. Shen, B. Wang, H. Lai, J. Zhu, Z. Hu, J. Yin, Towards multi-pose guided virtual try-on network, in: Proceedings of the IEEE/CVF International Conference on Computer Vision, 2019, pp. 90269035.

[3] X. Han, Z. Wu, Z. Wu, R. Yu, L. S. Davis, Viton: An image-based virtual try-on network, in: Proceedings of the IEEE conference on computer vision and pattern recognition, 2018, pp. 7543-7552.

[4] N. Jetchev, U. Bergmann, The conditional analogy gan: Swapping fashion articles on people images, in: Proceedings of the IEEE International Conference on Computer Vision Workshops, 2017, pp. 2287-2292.

[5] M. Lewis, S. Varadharajan, I. Kemelmacher-Shlizerman, Tryongan: Bodyaware try-on via layered interpolation, ACM Transactions on Graphics (TOG) 40 (4) (2021) 1-10

[6] S. Zhu, R. Urtasun, S. Fidler, D. Lin, C. Change Loy, Be your own prada: Fashion synthesis with structural coherence, in: Proceedings of the IEEE international conference on computer vision, 2017, pp. 1680-1688.
[7] B. Wang, H. Zheng, X. Liang, Y. Chen, L. Lin, M. Yang, Toward characteristic-preserving image-based virtual try-on network, in: Proceedings of the European Conference on Computer Vision (ECCV), 2018, pp. 589-604.

[8] H. Yang, R. Zhang, X. Guo, W. Liu, W. Zuo, P. Luo, Towards photorealistic virtual try-on by adaptively generating-preserving image content, in: Proceedings of the IEEE/CVF Conference on Computer Vision and Pattern Recognition, 2020, pp. 7850-7859

[9] Y. Men, Y. Mao, Y. Jiang, W.-Y. Ma, Z. Lian, Controllable person image synthesis with attribute-decomposed gan, in: Proceedings of the IEEE/CVF Conference on Computer Vision and Pattern Recognition, 2020, pp. 50845093.

[10] A. Neuberger, E. Borenstein, B. Hilleli, E. Oks, S. Alpert, Image based virtual try-on network from unpaired data, in: Proceedings of the IEEE/CVF Conference on Computer Vision and Pattern Recognition, 2020, pp. 51845193

[11] K. Sarkar, D. Mehta, W. Xu, V. Golyanik, C. Theobalt, Neural rerendering of humans from a single image, in: European Conference on Computer Vision, Springer, 2020, pp. 596-613.

[12] K. Sarkar, V. Golyanik, L. Liu, C. Theobalt, Style and pose control for image synthesis of humans from a single monocular view, arXiv preprint arXiv:2102.11263 (2021).

[13] A. Raj, P. Sangkloy, H. Chang, J. Lu, D. Ceylan, J. Hays, Swapnet: Garment transfer in single view images, in: Proceedings of the European Conference on Computer Vision (ECCV), 2018, pp. 666-682.

[14] T. Karras, S. Laine, T. Aila, A style-based generator architecture for generative adversarial networks, in: Proceedings of the IEEE/CVF Conference on Computer Vision and Pattern Recognition, 2019, pp. 4401-4410.

[15] O. Ronneberger, P. Fischer, T. Brox, U-net: Convolutional networks for biomedical image segmentation, in: International Conference on Medical image computing and computer-assisted intervention, Springer, 2015, pp. 234241.

[16] I. Goodfellow, J. Pouget-Abadie, M. Mirza, B. Xu, D. Warde-Farley, S. Ozair, A. Courville, Y. Bengio, Generative adversarial nets, Advances in neural information processing systems 27 (2014).

[17] D. P. Kingma, M. Welling, Auto-encoding variational bayes, in: International Conference on Learning Representations (ICLR), 2014.

[18] K. Regmi, A. Borji, Cross-view image synthesis using conditional gans, in: Proceedings of the IEEE Conference on Computer Vision and Pattern Recognition, 2018, pp. 3501-3510.

[19] P. Isola, J.-Y. Zhu, T. Zhou, A. A. Efros, Image-to-image translation with conditional adversarial networks, in: Proceedings of the IEEE conference on computer vision and pattern recognition, 2017, pp. 1125-1134.

[20] S. Gu, J. Bao, H. Yang, D. Chen, F. Wen, L. Yuan, Mask-guided portrait editing with conditional gans, in: Proceedings of the IEEE/CVF Conference on Computer Vision and Pattern Recognition, 2019, pp. 3436-3445.

[21] T.-C. Wang, M.-Y. Liu, J.-Y. Zhu, A. Tao, J. Kautz, B. Catanzaro, Highresolution image synthesis and semantic manipulation with conditional gans, in: Proceedings of the IEEE conference on computer vision and pattern recognition, 2018, pp. 8798-8807.

[22] N. Jetchev, U. Bergmann, The conditional analogy gan: Swapping fashion articles on people images, in: Proceedings of the IEEE International Conference on Computer Vision Workshops, 2017, pp. 2287-2292.

[23] W. Xu, C. Long, R. Wang, G. Wang, Drb-gan: A dynamic resblock generative adversarial network for artistic style transfer, in: Proceedings of the IEEE/CVF International Conference on Computer Vision, 2021, pp. 63836392.

[24] S. Kim, J. Do, M. Kim, Pseudo-supervised learning for semantic multistyle transfer, IEEE Access 9 (2021) 7930-7942. 
[25] K. Sarkar, V. Golyanik, L. Liu, C. Theobalt, Style and pose control for image synthesis of humans from a single monocular view, arXiv preprint arXiv:2102.11263 (2021)..

[26] M. Wang, G.-Y. Yang, R. Li, R.-Z. Liang, S.-H. Zhang, P. M. Hall, S.$\mathrm{M}$. Hu, Example-guided style-consistent image synthesis from semantic labeling, in: Proceedings of the IEEE/CVF Conference on Computer Vision and Pattern Recognition, 2019, pp. 1495-1504.

[27] P. Zhu, R. Abdal, Y. Qin, P. Wonka, Sean: Image synthesis with semantic region-adaptive normalization, in: Proceedings of the IEEE/CVF Conference on Computer Vision and Pattern Recognition, 2020, pp. 5104-5113.

[28] T. Park, M.-Y. Liu, T.-C. Wang, J.-Y. Zhu, Semantic image synthesis with spatially-adaptive normalization, in: Proceedings of the IEEE/CVF Conference on Computer Vision and Pattern Recognition, 2019, pp. 23372346.

[29] B. Zhang, M. He, J. Liao, P. V. Sander, L. Yuan, A. Bermak, D. Chen, Deep exemplar-based video colorization, in: Proceedings of the IEEE/CVF Conference on Computer Vision and Pattern Recognition, 2019, pp. 80528061.

[30] X. Liang, K. Gong, X. Shen, L. Lin, Look into person: Joint body parsing $\&$ pose estimation network and a new benchmark, IEEE transactions on pattern analysis and machine intelligence 41 (4) (2018) 871-885.

[31] M. Omran, C. Lassner, G. Pons-Moll, P. Gehler, B. Schiele, Neural body fitting: Unifying deep learning and model based human pose and shape estimation, in: 2018 international conference on 3D vision (3DV), IEEE, 2018, pp. 484-494.

[32] Z. Liu, P. Luo, S. Qiu, X. Wang, X. Tang, Deepfashion: Powering robust clothes recognition and retrieval with rich annotations, in: Proceedings of the IEEE conference on computer vision and pattern recognition, 2016, pp. 10961104.

[33] K. Gong, X. Liang, D. Zhang, X. Shen, L. Lin, Look into person: Selfsupervised structure-sensitive learning and a new benchmark for human parsing, in: Proceedings of the IEEE Conference on Computer Vision and Pattern Recognition, 2017, pp. 932-940.

[34] C. Lassner, J. Romero, M. Kiefel, F. Bogo, M. J. Black, P. V. Gehler, Unite the people: Closing the loop between $3 \mathrm{~d}$ and $2 \mathrm{~d}$ human representations, in: Proceedings of the IEEE conference on computer vision and pattern recognition, 2017, pp. 6050-6059.

[35] K. He, X. Zhang, S. Ren, J. Sun, Deep residual learning for image recognition, in: Proceedings of the IEEE conference on computer vision and pattern recognition, 2016, pp. 770-778.

[36] M. Loper, N. Mahmood, J. Romero, G. Pons-Moll, M. J. Black, Smpl: A skinned multi-person linear model, ACM transactions on graphics (TOG) 34 (6) (2015) 1-16.

[37] P. Zhang, B. Zhang, D. Chen, L. Yuan, F. Wen, Cross-domain correspondence learning for exemplar-based image translation, in: Proceedings of the IEEE/CVF Conference on Computer Vision and Pattern Recognition, 2020, pp. 5143-5153.

[38] Z. Wang, A. C. Bovik, H. R. Sheikh, E. P. Simoncelli, Image quality assessment: from error visibility to structural similarity, IEEE transactions on image processing 13 (4) (2004) 600-612.

[39] A. Hore, D. Ziou, Image quality metrics: Psnr vs. ssim, in: 2010 20th international conference on pattern recognition, IEEE, 2010, pp. 2366-2369. 